\title{
Effects of Intraoperative Lung-Protective Ventilation on Clinical Outcomes in Patients With Traumatic Brain Injury: A Randomized Controlled Trial
}

\section{Lulu Jiang}

Second Xiangya Hospital of Central South University

\section{Yujuan Wu}

Xiangtan Central Hospital

\section{Yang Zhang}

Northern Jiangsu People's Hospital

Dahao Lu

Northern Jiangsu People's Hospital

Keshi Yan

Northern Jiangsu People's Hospital

Ju Gao ( $\boldsymbol{1 7 8 2 0 1 0 4 9 @ c s u . e d u . c n ~ ) ~}$

Northern Jiangsu People's Hospital

\section{Research Article}

Keywords: Traumatic brain injury, Lung-protective ventilation, Postoperative pulmonary complications, Optic nerve sheath diameter, Glial fibrillary acidic protein, Ubiquitin carboxyl-terminal hydrolase isozyme L1

Posted Date: January 4th, 2021

DOl: https://doi.org/10.21203/rs.3.rs-128785/v1

License: (a) (1) This work is licensed under a Creative Commons Attribution 4.0 International License. Read Full License 


\section{Abstract}

Background: Secondary lung injury is the most common non-neurological complication after traumatic brain injury (TBI). Lung-protective ventilation (LPV) has been proven to improve perioperative oxygenation and lung compliance in some critical patients. This study aimed to investigate whether intraoperative LPV could improve respiratory function and prevent postoperative complications in emergency TBI patients.

Methods: Ninety TBI patients were randomly allocated to three groups (1:1:1): Group A, conventional mechanical ventilation [tidal volume (VT) $10 \mathrm{~mL} / \mathrm{kg}$ only]; Group B, small VT $(8 \mathrm{~mL} / \mathrm{kg})+$ positive endexpiratory pressure (PEEP) $\left(5 \mathrm{cmH}_{2} \mathrm{O}\right)$; Group C, small VT $(8 \mathrm{~mL} / \mathrm{kg})+\operatorname{PEEP}\left(5 \mathrm{cmH}_{2} \mathrm{O}\right)+$ recruitment maneuvers (RMs). Primary outcomes were intraoperative respiratory mechanics parameters and incidences of postoperative pulmonary complications; Secondary outcomes were serum levels of brain injury markers and incidences of postoperative neurological complications.

Results: Seventy-nine patients completed final analysis. Intraoperative $\mathrm{PaO}_{2}$ and dynamic pulmonary compliance of Group B and C were higher than those of Group A ( $P=0.028 ; P=0.005)$, while their airway peak pressure and plateau pressure were lower than those of group $\mathrm{A}(P=0.004 ; P=0.005)$. Compared to Group A, postoperative 30-day incidences of hypoxemia, pulmonary infection and atelectasis of the other two groups significantly decreased $(52.0 \%$ vs. $14.3 \%$ vs. $19.2 \%, P=0.005 ; 84.0 \%$ vs. $50.0 \%$ vs. $42.3 \%$, $P=0.006 ; 24.0 \%$ vs. $3.6 \%$ vs. $0.0 \%, P=0.004)$. Moreover, intraoperative hypotension in Group $C$ was more frequent than that in Group $A$ and $B(P=0.007)$. At the end of surgery, serum levels of glial fibrillary acidic protein and ubiquitin carboxyl-terminal hydrolase isozyme $L 1$ in Group $B$ were lower than those in group $A$ and $C(P=0.002 ; P<0.001)$. Postoperative incidences of neurological complications among three groups were comparable.

Conclusions: Intraoperative continuous administration of small VT + PEEP is beneficial to TBI patients. Additional RMs with caution can be performed to prevent the disturbance of the stability of cerebral hemodynamics.

Trial registration: Chinese Clinical Trial Registry (ChiCTR2000038314), retrospectively registered on September 17, 2020.

\section{Background}

Traumatic brain injury (TBI) is a major medical and socioeconomic problem. Over 50 million people worldwide develop TBI every year, and the morbidity has increased in the past decade [1]. TBI causes a wide range of systemic effects. It was reported that $89 \%$ of severe TBI patients experienced at least one non-neurological complication, of which $81 \%$ developed respiratory dysfunction, involving $23 \%$ of respiratory failure cases. Hence, respiratory complications were prevalent non-neurological disorders after TBI [2]. Besides, neural and humoral regulation after injury leads to an attenuated response of lung 
tissues to stress $[3,4]$, thus increasing the risk of pulmonary complications, especially pulmonary infection, neurogenic pulmonary edema (NPE), ventilator-associated lung injury (VALI), and atelectasis.

Tidal volume (VT) in general anesthesia is usually set $10-15 \mathrm{~mL} / \mathrm{kg}$ corrected body weight (CBW), which is higher than that of most mammals with spontaneous respiration. High VT ventilation may cause alveolar overdistention, inflammatory mediator spillover, and VALI. Now, lung-protective ventilation (LPV) is defined as VT $\leq 8 \mathrm{~mL} / \mathrm{kg}$, positive end-expiratory pressure (PEEP) $\geq 5 \mathrm{cmH}_{2} \mathrm{O}$, and airway plateau pressure (Pplat) $\leq 30 \mathrm{cmH}_{2} \mathrm{O}$, which is recognized as the optimal ventilation mode for patients with acute respiratory distress syndrome (ARDS) in the intensive care unit (ICU) [5]. In view of satisfactory practices of LPV in ARDS patients, perioperative lung protection in operating room has also been highlighted by more anesthesiologists.

Various factors attribute to the complex interactions between mechanical ventilation (MV) and cerebral hemodynamics during operation. Plenty of clinical trials or meta-analysis have found that perioperative application of LPV can improve intraoperative oxygenation and lung compliance, and relieve postoperative pulmonary complications (PPCs). However, PEEP or recruitment maneuvers (RMs) may disrupt the stability of cerebral hemodynamics in emergency TBI patients, who are often excluded from these studies. Whether perioperative LPV is also beneficial to these patients requires explorations. A recent retrospective study involving 28,644 TBI patients in ICU showed no significant changes in intracranial pressure (ICP) and cerebral perfusion pressure (CPP) after applying LPV, indicating its safety of respiratory support in TBI patients [6].

Here, we conducted a randomized controlled trial with the hypothesis that intraoperative use of LPV can improve respiratory function and prevent postoperative complications in TBI patients. The primary aim was to assess intraoperative respiratory mechanics parameters and incidences of PPCs in TBI patients intervened with LPV. Secondary aims investigated their serum levels of brain injury markers and incidences of postoperative neurological complications.

\section{Methods}

\section{Study Design, approvals and registration}

A single-center, randomized controlled study involving 90 TBI patients was approved by the Ethics Committee of Northern Jiangsu People's Hospital (2019113). Informed consent was obtained from patients or their relatives. The trial was retrospectively registered at Chinese Clinical Trial Registry (ChiCTR2000038314) on 17/09/2020.

\section{Patients}

TBI patients aging 18-65 years who underwent emergency intracranial evacuation of hematoma were enrolled. No limitation in gender was set. Their body mass index (BMI) ranged from 18.5 to $29.9 \mathrm{~kg} / \mathrm{m}^{2}$ with the American Society of Anesthesiologists (ASA) Classification $\Downarrow$ or $\rrbracket$. 
Patients with a history of mental diseases or other neurological disorders (epilepsy, dementia, cerebrovascular malformation, etc.), severe cardiovascular diseases (valvular heart disease, pericarditis, cor pulmonale, etc.), and severe hepatic or renal insufficiency (cirrhosis, chronic renal failure, nephrotic syndrome, etc.), were excluded. Patients who had stroke, myocardial infarction or major surgery within three months and refused to participate were also excluded.

Patients were assigned by computer-generated randomized sequence to three groups (1:1:1): Group $A$ (conventional MV), Group B (small VT $+5 \mathrm{cmH}_{2} \mathrm{O}$ PEEP), Group C (small VT $+5 \mathrm{cmH}_{2} \mathrm{O}$ PEEP $+\mathrm{RMs}$ ). The random allocation scheme was sealed by the principal investigator in opaque envelopes. Two experienced anesthesiologists blinded to the random allocation enrolled eligible participants, and the other two assigned participants to interventions.

\section{Anesthesia}

Midazolam (0.05 mg/kg), sufentanil (0.5ug/kg), propofol (1-2 mg/kg), cis-atracurium (0.15$0.20 \mathrm{mg} / \mathrm{kg}$ ) were intravenously injected for anesthesia induction. After tracheal intubation, the anesthesia machine was connected to start MV.

Inhalation of $2 \%$ sevoflurane during operation, and intravenous pump injection of remifentanil (0.1$0.3 \mu \mathrm{g} / \mathrm{kg} / \mathrm{min})$, dexmedetomidine $(0.01 \mu \mathrm{g} / \mathrm{kg} / \mathrm{min})$, cis-atracurium ( $5 \mu \mathrm{g} / \mathrm{kg} / \mathrm{min}$ ) were performed for anesthesia maintenance, the dose of which was adjusted according to the depth of anesthesia. Vasoactive drugs were applied if cyclic fluctuations occurred during surgery.

\section{Mechanical ventilation}

All patients received MV after tracheal intubation under the same general anesthesia management. Immediately after intubation, volume-controlled ventilation was applied in three groups with VT $10 \mathrm{~mL} / \mathrm{kg}$ CBW, inspiration/expiration 1:2, fraction of inspired oxygen $100 \%$, oxygen flow $2 \mathrm{~L} / \mathrm{min}$, no PEEP and RMs. Five minutes later, the parameters of Group B and C were adjusted to $8 \mathrm{~mL} / \mathrm{kg} \mathrm{CBW}$ of VT and $5 \mathrm{cmH}_{2} \mathrm{O}$ PEEP continuously. Patients in Group $\mathrm{C}$ received twice RMs before opening and after closing the endocranium. Briefly, RMs were performed to maintain the airway pressure $30 \mathrm{cmH}_{2} \mathrm{O}$ for $30 \mathrm{~s}$. During operation, respiratory rate was adjusted according to arterial blood gas analysis to maintain end-tidal carbon dioxide partial pressure $\left(\mathrm{P}_{\mathrm{ET}} \mathrm{CO}_{2}\right)$ at $30-35 \mathrm{mmHg}$.

Removal of the tracheal catheter postoperatively was discussed by the anesthesiologist and the attending physician. When patients recovered spontaneous breath, swallow, cough reflex, VT $>6 \mathrm{~mL} / \mathrm{kg}$, oxygen saturation $\left(\mathrm{SpO}_{2}\right)>95 \%$ for $10 \mathrm{~min}$, a gentle suction to clean the tube and oropharyngeal secretions, removal of the tracheal catheter, and delivery of oxygen by mask with $5 \mathrm{~L} / \mathrm{min}$ were performed. The mask was removed 10 min later, followed by 20 -min observation and then patients were sent to the ward. Others with tracheal catheter were directly sent to ICU in the case of sedation, analgesia and ventilator.

\section{Outcomes}


Primary outcomes were: 1) intraoperative oxygenation and respiratory mechanics parameters [arterial partial pressure of oxygen $\left(\mathrm{PaO}_{2}\right)$, arterial partial pressure of carbon dioxide $\left(\mathrm{PaCO}_{2}\right)$, pulmonary dynamic compliance (Cdyn), airway peak pressure (Ppeak), Pplat, heart rate (HR), mean arterial pressure (MAP)]; 2) the incidences of intraoperative pulmonary and cardiovascular adverse reactions $\left[\mathrm{SpO}_{2}<90 \%\right.$ or $\mathrm{P}_{\mathrm{ET}} \mathrm{CO}_{2}$ $>45 \mathrm{mmHg}$ or systolic blood pressure (SBP) $<90 \mathrm{mmHg}$ for more than $1 \mathrm{~min}$, any arrhythmia]; 3) the incidences of postoperative 30-day pulmonary complications (hypoxemia, pulmonary infection, atelectasis, ARDS, VALI, NPE).

Secondary outcomes were intraoperative serum levels of brain injury markers [glial fibrillary acidic protein (GFAP), ubiquitin carboxyl-terminal hydrolase isozyme L1 (UCHL1)]and the incidences of postoperative 30-day neurological complications (intracranial infection, intracranial hypertension, epilepsy, encephaledema, reoperation).

Other outcomes included intraoperative optic nerve sheath diameter (ONSD), postoperative duration of MV, length of stay, 30-day Glasgow Outcome Scale Extended (GOSE).

\section{Data collection}

The baseline characteristics were sex, age, BMI, ASA class, preoperative Glasgow Coma Scale (GCS) and hemoglobin concentration, intraoperative bleeding volume and infusion quantity, total operative and anesthesia time.

Blood gas analysis was performed on $1 \mathrm{~mL}$ radial artery blood sample at the onset of $M V\left(T_{1}\right)$, ventilation for $60 \mathrm{~min}\left(\mathrm{~T}_{2}\right)$, and the end of surgery $\left(\mathrm{T}_{3}\right)$. The ONSD was measured by color Doppler ultrasound at anesthesia induction $\left(T_{0}\right), T_{1}$, after applying PEEP $\left(t_{0}\right)$, before the first RM $\left(t_{1}\right)$, after the first RM $\left(t_{2}\right)$, before the second RM $\left(t_{3}\right)$, after the second $R M\left(t_{4}\right)$ and $T_{3} .5 \mathrm{~mL}$ internal jugular vein blood sample of each patient at $T_{1}, T_{2}$ and $T_{3}$ was placed in vacuum blood collection tubes, whose supernatant was collected and detected by ELISA Kit of GFAP and UCHL1 (ab223867, Abcam; CY-8092, CircuLex) according to corresponding instructions.

\section{Statistical analysis}

Sample size was calculated as previously reported [7] and our pilot trial. According to the calculation formula for comparison of multiple sample rates [8], 24 patients per group were needed to detect a significant change in the incidence of PPC after applying LPV, with type $\nabla$ error of $0.05,90 \%$ power and dropout rate of $20 \%$.

Statistical Package for the Social Sciences version 22.0 was used for data processing. Normally distributed measurement data were expressed as mean \pm standard deviation, and Levene test was conducted for assessing the homogeneity. If the data met the hypothesis of equal variance, StudentNewman-Keuls was applied to compare differences between any two samples; Otherwise, after performing Kruskal-Wallis $\mathrm{H}$ test, Bonferroni method was utilized to correct the significance level for posthoc multiple comparison. Measurement data of skew distribution were expressed as median and 
interquartile range, which were compared in the same way as data with unequal variances. Enumeration data expressed as percentages were analyzed by Chi-square test for $\mathrm{R} \times \mathrm{C}$ table data. Pairwise comparison was conducted if all theoretical frequencies were greater than 5 ; Otherwise, Fisher exact probability test and pairwise comparisons were conducted. $P<0.05$ was considered statistically significant.

\section{Results}

From December 2019 to September 2020, we recruited 90 eligible TBI patients and equally assigned them to conventional MV (Group A), small VT $+5 \mathrm{cmH}_{2} \mathrm{O}$ PEEP (Group B), small VT $+5 \mathrm{cmH}_{2} \mathrm{O}$ PEEP $+\mathrm{RMs}$ (Group C). Finally, there were 79 participants completed final analysis, as 5, 2 and 4 patients died within postoperative 30 days in Group A, B and C, respectively (Fig. 1). Baseline characteristics of participants were comparable (Table 1). 
Table 1

Baseline characteristics by randomized group

\begin{tabular}{|c|c|c|c|c|}
\hline & $\begin{array}{l}\text { Group A }(n= \\
25)\end{array}$ & $\begin{array}{l}\text { Group B }(n= \\
28)\end{array}$ & $\begin{array}{l}\text { Group } C(n= \\
26)\end{array}$ & $P$ \\
\hline \multicolumn{5}{|l|}{ Sex, n (\%) } \\
\hline Male & $17(68.0)$ & $20(71.4)$ & $20(76.9)$ & \multirow[t]{2}{*}{0.772} \\
\hline Female & $8(32.0)$ & $8(28.6)$ & $6(23.1)$ & \\
\hline Age, years, median (IQR) & $\begin{array}{l}55.0(45.0- \\
60.0)\end{array}$ & $\begin{array}{l}52.5(45.3- \\
56.0)\end{array}$ & $\begin{array}{l}50.0(43.5- \\
56.0)\end{array}$ & 0.500 \\
\hline $\mathrm{BMI}, \mathrm{kg} / \mathrm{m}^{2}$, mean $\pm \mathrm{SD}$ & $23.3 \pm 2.1$ & $22.9 \pm 1.8$ & $22.6 \pm 1.8$ & 0.469 \\
\hline \multicolumn{5}{|l|}{ ASA Class, n (\%) } \\
\hline ० & $9(36.0)$ & $7(25.0)$ & $7(26.9)$ & \multirow[t]{2}{*}{0.649} \\
\hline ૧ & $16(64.0)$ & $21(75.0)$ & $19(73.1)$ & \\
\hline \multicolumn{5}{|l|}{ Glasgow Coma Scale, n (\%) ${ }^{a}$} \\
\hline $13-15$ & $1(4.0)$ & $3(10.7)$ & $3(11.5)$ & \multirow[t]{3}{*}{0.911} \\
\hline $9-12$ & $9(36.0)$ & $9(32.1)$ & $8(30.8)$ & \\
\hline$\leq 8$ & $15(60.0)$ & $16(57.1)$ & $15(57.7)$ & \\
\hline $\begin{array}{l}\text { Preoperative hemoglobin, } \mathrm{g} / \mathrm{dL} \text {, } \\
\text { median (IQR) }\end{array}$ & $\begin{array}{l}12.0(10.0- \\
13.0)\end{array}$ & $\begin{array}{l}13.0(11.3- \\
13.8)\end{array}$ & $\begin{array}{l}12.0(11.0- \\
13.3)\end{array}$ & 0.462 \\
\hline $\begin{array}{l}\text { Intraoperative amount of bleeding, } \mathrm{mL} \text {, } \\
\text { median (IQR) }\end{array}$ & $\begin{array}{l}300.0(200.0- \\
400.0)\end{array}$ & $\begin{array}{l}300.0(200.0- \\
437.5)\end{array}$ & $\begin{array}{l}300.0(200.0- \\
500.0)\end{array}$ & 0.440 \\
\hline \multirow{2}{*}{$\begin{array}{l}\text { Intraoperative fluid infusion volume, } \\
\text { mL, median (IQR) }\end{array}$} & 2500.0 & 2500.0 & 2500.0 & \multirow[t]{2}{*}{0.810} \\
\hline & $\begin{array}{l}(1975.0- \\
3175.0)\end{array}$ & $\begin{array}{l}(2000.0- \\
3000.0)\end{array}$ & $\begin{array}{l}(2000.0- \\
3000.0)\end{array}$ & \\
\hline Operative time, min, median (IQR) & $\begin{array}{l}200.0(150.0- \\
237.5)\end{array}$ & $\begin{array}{l}177.5(156.3- \\
199.5)\end{array}$ & $\begin{array}{l}162.5(150.0- \\
222.5)\end{array}$ & 0.379 \\
\hline Anesthesia time, min, median (IQR) & $\begin{array}{l}245.0(200.0- \\
285.0)\end{array}$ & $\begin{array}{l}220.0(201.3- \\
253.8)\end{array}$ & $\begin{array}{l}202.5(193.8- \\
270.0)\end{array}$ & 0.508 \\
\hline \multicolumn{5}{|c|}{$\begin{array}{l}\text { ASA, American Society of Anesthesiologists; BMI, body mass index; IQR, interquartile range; SD, } \\
\text { standard deviation. }\end{array}$} \\
\hline
\end{tabular}


Figure 2 showed the timeline of intraoperative LPV strategy. Table 2 summarized intraoperative blood gas analysis, respiratory mechanics and hemodynamics. At $\mathrm{T}_{1}$, no significant differences in $\mathrm{PaO}_{2}, \mathrm{PaCO}_{2}$, Cdyn, Ppeak and Pplat were detected among three groups. At $\mathrm{T}_{2}$, compared to Group $\mathrm{A}$, the median $\mathrm{PaO}_{2}$ and Cdyn increased significantly in Group $B$ and $C(336.0$ vs. 375.5 vs. $388.0 \mathrm{mmHg}, P=0.028 ; 320.0$ vs. 360.0 vs. $\left.350.0 \mathrm{~mL} / \mathrm{cmH}_{2} \mathrm{O}, P=0.005\right)$, while their median Ppeak and Pplat decreased significantly (18.0 vs. 17.0 vs. $17.0 \mathrm{cmH}_{2} \mathrm{O}, P=0.004 ; 14.0$ vs. 13.0 vs. $\left.13.0 \mathrm{cmH}_{2} \mathrm{O}, P=0.005\right)$. No significant difference in $\mathrm{PaCO}_{2}$ was detected. At $\mathrm{T}_{3}$, the median $\mathrm{PaO}_{2}, \mathrm{PaCO}_{2}$, Cdyn in Group $\mathrm{B}$ and $\mathrm{C}$ were higher than those in Group A (340.0 vs. 397.5 vs. 402.5 mmHg, $P=0.005 ; 40.0$ vs. 44.0 vs. 42.0 mmHg, $P=0.025 ; 330.0$ vs. 340.0 vs. $\left.340.0 \mathrm{~mL} / \mathrm{cmH}_{2} \mathrm{O}, P=0.009\right)$, which were opposite to the median Ppeak and Pplat (19.0 vs. 17.0 vs. $17.0 \mathrm{cmH}_{2} \mathrm{O}, P=0.012 ; 16.0$ vs. 13.0 vs. $\left.14.0 \mathrm{cmH}_{2} \mathrm{O}, P=0.003\right)$. There were no significant differences in aforementioned indicators between Group $B$ and $C$ both at $T_{2}$ and $T_{3}$. Meanwhile, HR and MAP among three groups were comparable throughout the surgery. 
Table 2

Intraoperative blood gas analysis, respiratory mechanics and hemodynamics

\begin{tabular}{|c|c|c|c|c|c|}
\hline & & Group A $(n=25)$ & Group B $(n=28)$ & Group C $(n=26)$ & $P$ \\
\hline \multirow[t]{3}{*}{$\mathrm{PaO}_{2}, \mathrm{mmHg}$} & $\mathrm{T}_{1}$ & $\begin{array}{l}419.0(381.5- \\
486.0)\end{array}$ & $426.0(400.8-450.3)$ & 433.5 (367.5-487.3) & 0.781 \\
\hline & $\mathrm{T}_{2}$ & $\begin{array}{l}436.0(382.0- \\
487.0)\end{array}$ & $475.5(447.3-496.8)^{*}$ & $\begin{array}{l}488.0(409.8- \\
527.3)^{\star}\end{array}$ & 0.028 \\
\hline & $\mathrm{T}_{3}$ & $\begin{array}{l}440.0(394.5- \\
493.5)\end{array}$ & $\begin{array}{l}497.5(486.8- \\
534.5)^{\#}\end{array}$ & $\begin{array}{l}502.5(441.3- \\
554.0)^{\star}\end{array}$ & 0.005 \\
\hline \multirow[t]{3}{*}{$\mathrm{PaCO}_{2}, \mathrm{mmHg}$} & $\mathrm{T}_{1}$ & $45.0(39.5-50.0)$ & $46.0(40.3-49.0)$ & $46.0(37.0-49.0)$ & 0.881 \\
\hline & $\mathrm{T}_{2}$ & $42.0(39.0-46.0)$ & $42.0(40.0-47.8)$ & $42.0(38.8-47.3)$ & 0.970 \\
\hline & $\mathrm{T}_{3}$ & $40.0(38.0-42.0)$ & $44.0(39.3-47.8)^{\star}$ & $42.0(39.0-46.3)^{*}$ & 0.025 \\
\hline \multirow[t]{3}{*}{$\begin{array}{l}\text { Cdyn, } \\
\mathrm{mL} / \mathrm{cmH}_{2} \mathrm{O}\end{array}$} & $\mathrm{T}_{1}$ & $\begin{array}{l}320.0(300.0- \\
335.0)\end{array}$ & $320.0(310.0-350.0)$ & $310.0(300.0-322.5)$ & 0.080 \\
\hline & $\mathrm{T}_{2}$ & $\begin{array}{l}320.0(295.0- \\
355.0)\end{array}$ & $\begin{array}{l}360.0(332.5- \\
370.0)^{\#}\end{array}$ & $\begin{array}{l}350.0(340.0- \\
360.0)^{\star}\end{array}$ & 0.005 \\
\hline & $\mathrm{T}_{3}$ & $\begin{array}{l}330.0(305.0- \\
345.0)\end{array}$ & $340.0(330.0-360.0)^{*}$ & $\begin{array}{l}340.0(330.0- \\
370.0)^{\star}\end{array}$ & 0.009 \\
\hline \multirow[t]{3}{*}{ Ppeak, $\mathrm{cmH}_{2} \mathrm{O}$} & $\mathrm{T}_{1}$ & $17.0(16.0-20.0)$ & $18.0(16.0-19.0)$ & $19.0(17.0-20.0)$ & 0.379 \\
\hline & $\mathrm{T}_{2}$ & $18.0(17.5-21.0)$ & $17.0(16.0-18.8)^{\#}$ & $17.0(15.8-19.0)^{*}$ & 0.004 \\
\hline & $\mathrm{T}_{3}$ & $19.0(18.0-21.0)$ & $17.0(15.3-20.0)^{\star}$ & $17.0(16.0-19.0)^{*}$ & 0.012 \\
\hline \multirow[t]{3}{*}{ Pplat, $\mathrm{cmH}_{2} \mathrm{O}$} & $\mathrm{T}_{1}$ & $13.0(11.5-14.5)$ & $14.0(12.0-15.8)$ & $15.0(13.0-16.3)$ & 0.068 \\
\hline & $\mathrm{T}_{2}$ & $14.0(13.0-17.0)$ & $13.0(11.3-14.8)^{*}$ & $13.0(11.0-15.0)^{\star}$ & 0.005 \\
\hline & $\mathrm{T}_{3}$ & $16.0(13.5-17.0)$ & $13.0(11.0-15.0)^{\#}$ & $14.0(11.8-15.3)^{*}$ & 0.003 \\
\hline${ }_{1}^{\text {Heart rate, } \mathrm{min}^{-}}$ & $\mathrm{T}_{1}$ & $81(65-102)$ & $80(65-93)$ & $76(72-92)$ & 0.779 \\
\hline
\end{tabular}

Data are presented as median (interquartile range).

Cdyn, pulmonary dynamic compliance; DBP, diastolic blood pressure; MAP, mean arterial pressure; $\mathrm{PaCO}_{2}$, arterial partial pressure of carbon dioxide; $\mathrm{PaO}_{2}$, arterial partial pressure of oxygen; Ppeak, airway peak pressure; Pplat, airway plateau pressure; SBP, systolic blood pressure.

a $M A P=(S B P+D B P * 2) / 3$.

${ }^{*} P<0.05,{ }^{\#} P<0.01$ compared to Group A at the same point in time. 


\begin{tabular}{|c|c|c|c|c|c|}
\hline & & Group A $(n=25)$ & Group B $(n=28)$ & Group C $(n=26)$ & $P$ \\
\hline \multirow{5}{*}{ MAP, mmHg ${ }^{a}$} & $\mathrm{~T}_{2}$ & $66(58-92)$ & $70(62-87)$ & $68(62-83)$ & 0.891 \\
\hline & $T_{3}$ & $64(56-93)$ & $65(59-80)$ & $68(63-85)$ & 0.421 \\
\hline & $\mathrm{T}_{1}$ & $88.0(77.0-101.5)$ & $93.0(85.0-101.8)$ & $93.5(88.5-109.0)$ & 0.419 \\
\hline & $\mathrm{T}_{2}$ & $79.0(73.5-90.0)$ & $86.0(77.0-95.0)$ & $82.0(73.8-86.5)$ & 0.210 \\
\hline & $\mathrm{T}_{3}$ & $77.0(71.0-92.5)$ & $78.0(71.3-84.3)$ & $81.0(72.0-91.5)$ & 0.802 \\
\hline \multicolumn{6}{|c|}{ Data are presented as median (interquartile range). } \\
\hline \multicolumn{6}{|c|}{$\begin{array}{l}\mathrm{Cdyn}, \text { pulmonary dynamic compliance; } \mathrm{DBP} \text {, diastolic blood pressure; } \mathrm{MAP} \text {, mean arterial pressure; } \\
\mathrm{PaCO}_{2} \text {, arterial partial pressure of carbon dioxide; } \mathrm{PaO}_{2} \text {, arterial partial pressure of oxygen; } \mathrm{Ppeak} \text {, } \\
\text { airway peak pressure; Pplat, airway plateau pressure; } \mathrm{SBP} \text {, systolic blood pressure. }\end{array}$} \\
\hline \multicolumn{6}{|c|}{${ }^{\mathrm{a}} \mathrm{MAP}=(\mathrm{SBP}+\mathrm{DBP} * 2) / 3$} \\
\hline
\end{tabular}

Furthermore, intraoperative respiratory and cardiovascular adverse reactions were recorded and a 30-day postoperative follow-up was conducted (Table 3). Compared with Group A and B, the incidence of intraoperative hypotension (SBP $<90 \mathrm{mmHg})$ increased significantly in Group C $(32.0 \%$ vs. $39.3 \%$ vs. $73.1 \%, P=0.007$ ), whilst no significant differences in the incidences of arrhythmia, $\mathrm{SPO}_{2}<90 \%$ and $\mathrm{P}_{\mathrm{ET}} \mathrm{CO}_{2}>45 \mathrm{mmHg}$ were found among three groups. Our follow-up results showed that the incidences of hypoxemia, pulmonary infection and atelectasis in Group $B$ and $C$ were significantly lower than those in Group A $(52.0 \%$ vs. $14.3 \%$ vs. $19.2 \%, P=0.005 ; 84.0 \%$ vs. $50.0 \%$ vs. $42.3 \%, P=0.006 ; 24.0 \%$ vs. $3.6 \%$ vs. $0.0 \%, P=0.004)$. However, the incidences of ARDS, VALI and NPE among three groups were comparable. Besides, there were no significant differences in PPCs between Group B and C. Postoperative incidences of neurological complications of three groups were similar. The median postoperative ventilation time in Group A was significantly longer than that of Group B and C (72.0 vs. 24.0 vs. 24.0 h, $P=0.006)$, which was comparable between the latter two groups. Likewise, there were no significant differences in GOSE score and hospital stay in three groups. 
Table 3

Intraoperative adverse reactions and postoperative 30-day follow-up

\begin{tabular}{|c|c|c|c|c|}
\hline & $\begin{array}{l}\text { Group } A(n= \\
25)\end{array}$ & $\begin{array}{l}\text { Group B (n } \\
=28)\end{array}$ & $\begin{array}{l}\text { Group } C(n \\
=26)\end{array}$ & $P$ \\
\hline \multicolumn{5}{|l|}{ Intraoperative adverse reactions, n (\%) } \\
\hline $\mathrm{SpO}_{2}<90 \%$ & $2(8.0)$ & $1(3.6)$ & $1(3.8)$ & 0.685 \\
\hline $\mathrm{P}_{\mathrm{ET}} \mathrm{CO}_{2}>45 \mathrm{mmHg}$ & $1(4.0)$ & $5(17.9)$ & $5(19.2)$ & 0.213 \\
\hline $\mathrm{SBP}<90 \mathrm{mmHg}$ & $8(32.0)$ & $11(39.3)$ & $19(73.1)^{\mathrm{ab}}$ & 0.007 \\
\hline Arrhythmia & $2(8.0)$ & $5(17.9)$ & $4(15.4)$ & 0.609 \\
\hline \multicolumn{5}{|c|}{ Postoperative respiratory complications, n (\%) } \\
\hline Hypoxemia & $13(52.0)$ & $4(14.3)^{a}$ & $5(19.2)^{a}$ & 0.005 \\
\hline Pulmonary infection & $21(84.0)$ & $14(50.0)^{\mathrm{a}}$ & $11(42.3)^{a}$ & 0.006 \\
\hline Atelectasis & $6(24.0)$ & $1(3.6)^{\mathrm{a}}$ & $0(0.0)^{\mathrm{a}}$ & 0.004 \\
\hline Acute respiratory distress syndrome & $1(4.0)$ & $1(3.6)$ & $0(0.0)$ & 0.764 \\
\hline Ventilator-associated lung injury & $3(12.0)$ & $3(10.7)$ & $2(7.7)$ & 0.902 \\
\hline Neurogenic pulmonary edema & $1(4.0)$ & $0(0.0)$ & $1(3.9)$ & 0.537 \\
\hline \multicolumn{5}{|c|}{ Postoperative neurological complications, n (\%) } \\
\hline Intracranial infection & $3(12.0)$ & $3(10.7)$ & $4(15.4)$ & 0.915 \\
\hline Intracranial hypertension & $6(24.0)$ & $7(25.0)$ & $7(26.9)$ & 1.000 \\
\hline Epilepsy & $2(8.0)$ & $2(7.1)$ & $3(11.5)$ & 0.890 \\
\hline Encephaledema & $5(20.0)$ & $5(17.9)$ & $6(23.1)$ & 0.939 \\
\hline Reoperation & $2(8.0)$ & $1(3.6)$ & $2(7.7)$ & 0.733 \\
\hline \multicolumn{5}{|l|}{ Other } \\
\hline $\begin{array}{l}\text { Mechanical ventilation time, } \mathrm{h} \text {, median } \\
\text { (interquartile range) }\end{array}$ & $\begin{array}{l}72.0(36.0- \\
105.0)\end{array}$ & $\begin{array}{l}24.0(3.0- \\
62.0)^{\mathrm{a}}\end{array}$ & $\begin{array}{l}24.0(9.1- \\
66.0)^{\mathrm{a}}\end{array}$ & 0.006 \\
\hline
\end{tabular}

GOSE, Glasgow Outcome Scale Extended; $\mathrm{P}_{\mathrm{ET}} \mathrm{CO}_{2}$, end-tidal carbon dioxide partial pressure; SBP, systolic blood pressure; $\mathrm{SD}$, standard deviation; $\mathrm{SpO}_{2}$, oxygen saturation.

${ }^{\text {a }} P<0.05$ compared to Group A; ${ }^{b} P<0.05$ compared to Group B.

${ }^{c}$ GOSE is used to assess outcomes of patients with brain damage, which is divided into 8 levels. The higher the grade, the better the patient's prognosis. 


\begin{tabular}{|c|c|c|c|c|}
\hline & $\begin{array}{l}\text { Group } A(n= \\
25)\end{array}$ & $\begin{array}{l}\text { Group B (n } \\
=28)\end{array}$ & $\begin{array}{l}\text { Group C (n } \\
=26)\end{array}$ & $P$ \\
\hline Length of stay, days, mean \pm SD & $21.5 \pm 10.4$ & $21.9 \pm 8.3$ & $22.0 \pm 7.5$ & 0.975 \\
\hline GOSE score, mean $\pm \mathrm{SD}^{\mathrm{C}}$ & $5 \pm 1.6$ & $6 \pm 1.3$ & $5 \pm 1.2$ & 0.768 \\
\hline \multicolumn{5}{|c|}{$\begin{array}{l}\text { GOSE, Glasgow Outcome Scale Extended; } \mathrm{P}_{\mathrm{ET}} \mathrm{CO}_{2} \text {, end-tidal carbon dioxide partial pressure; } \mathrm{SBP} \text {, } \\
\text { systolic blood pressure; SD, standard deviation; } \mathrm{SpO}_{2} \text {, oxygen saturation. }\end{array}$} \\
\hline \multicolumn{5}{|c|}{ a $P<0.05$ compared to Group $A ;{ }^{b} P<0.05$ compared to Group B. } \\
\hline
\end{tabular}

As for ONSD (Table 4), there were no significant differences among three groups at $T_{0}, T_{1}$ and $T_{3}$. When compared within each group, the differences in Group A or B were comparable at any time. Comparisons in Group C were interesting. In detail, after performing each RM, the mean ONSD (mm) at $t_{2}$ or $t_{4}$ was not only significantly higher than that before carrying out each RM ( $t_{1} v s . t_{2}: 5.38 v s .5 .65 ; t_{3} v s . t_{4}: 5.38 v s$. $5.61 ; P<0.05)$, but it was higher than that at $\mathrm{T}_{0}$ or $\mathrm{T}_{3}\left(\mathrm{~T}_{0}\right.$ vs. $\mathrm{t}_{2}: 5.26$ vs. $5.65 ; \mathrm{T}_{0}$ vs. $\mathrm{t}_{4}: 5.26$ vs. $5.61 ; \mathrm{T}_{3}$ vs. $\mathrm{t}_{2}: 5.34$ vs. $5.65 ; \mathrm{T}_{3}$ vs. $\mathrm{t}_{4}: 5.34$ vs. $\left.5.61 ; P<0.05\right)$.

Table 4

Ultrasound measurement of ONSD

\begin{tabular}{|c|c|c|c|c|c|c|c|c|c|}
\hline & $\mathrm{T}_{0}$ & $\mathrm{~T}_{1}$ & $t_{0}$ & $t_{1}$ & $t_{2}$ & $t_{3}$ & $t_{4}$ & $T_{3}$ & $P$ \\
\hline $\begin{array}{l}\text { Group A } \\
(\mathrm{n}=25)\end{array}$ & $\begin{array}{l}5.32 \\
\pm 0.36\end{array}$ & $\begin{array}{l}5.37 \\
\pm 0.32\end{array}$ & NA & NA & NA & NA & NA & $\begin{array}{l}5.32 \\
\pm 0.30\end{array}$ & 0.864 \\
\hline $\begin{array}{l}\text { Group B } \\
(\mathrm{n}=28)\end{array}$ & $\begin{array}{l}5.33 \\
\pm 0.32\end{array}$ & $\begin{array}{l}5.37 \\
\pm 0.30\end{array}$ & $\begin{array}{l}5.44 \\
\pm 0.31\end{array}$ & NA & NA & NA & NA & $\begin{array}{l}5.38 \\
\pm 0.29\end{array}$ & 0.527 \\
\hline $\begin{array}{l}\text { Group C } \\
(n=28)\end{array}$ & $\begin{array}{l}5.26 \\
\pm 0.28\end{array}$ & $\begin{array}{l}5.32 \\
\pm 0.30\end{array}$ & $\begin{array}{l}5.37 \\
\pm 0.31\end{array}$ & $\begin{array}{l}5.38 \\
\pm 0.29\end{array}$ & $\begin{array}{l}5.65 \pm \\
0.28^{\mathrm{abcd}}\end{array}$ & $\begin{array}{l}5.38 \\
\pm 0.30\end{array}$ & $\begin{array}{l}5.61 \pm \\
0.28^{\mathrm{abcd}}\end{array}$ & $\begin{array}{l}5.34 \\
\pm 0.29\end{array}$ & $\begin{array}{l}<.001 \\
0.001\end{array}$ \\
\hline \multicolumn{10}{|c|}{ Data are presented as mean \pm standard deviation $(\mathrm{mm})$. } \\
\hline \multicolumn{10}{|c|}{ NA, not applicable; ONSD, optic nerve sheath diameter } \\
\hline
\end{tabular}

Table 5 showed serum levels of GFAP and UCHL1 in three groups at different time points. Both of them increased significantly in each group with the prolongation of operative time ( $P<0.001$, each). At $\mathrm{T}_{1}$, there were no significant differences in GFAP and UCHL1 levels among three groups. At $T_{2}$, the mean serum level of GFAP in Group B was the lowest (399.16 vs. 360.93 vs. $389.12 \mathrm{pg} / \mathrm{mL}, P=0.042)$, but significant difference was only detected between Group A and B. The mean serum level of UCHL1 in Group B was 
significantly lower than that of the other two groups (828.16 vs. $661.96 \mathrm{vs} .782 .00 \mathrm{pg} / \mathrm{mL}, P=0.001)$, which was comparable between Group A and C. Similarly, at $T_{3}$, the mean serum level of GFAP was significantly lower in Group B than the other groups (459.24 vs. 396.68 vs. $431.96 \mathrm{pg} / \mathrm{mL}, P=0.002)$, which was comparable in the latter two groups. The mean serum level of UCHL1 was the highest in Group A, which was the lowest in Group B (1223.00 vs. 849.21 vs. 1068.50 pg/mL, $P<0.001)$.

Table 5

Intraoperative serum levels of GFAP and UCHL1

\begin{tabular}{|c|c|c|c|c|c|}
\hline & & Group $A(n=25)$ & Group B $(n=28)$ & Group $C(n=26)$ & $P$ \\
\hline \multirow[t]{3}{*}{ GFAP } & $\mathrm{T}_{1}$ & $328.68 \pm 54.50$ & $325.79 \pm 55.82$ & $336.58 \pm 61.04$ & 0.776 \\
\hline & $\mathrm{T}_{2}$ & $399.16 \pm 55.40^{*}$ & $360.93 \pm 56.71^{a^{*}}$ & $389.12 \pm 57.32^{*}$ & 0.042 \\
\hline & $T_{3}$ & $459.24 \pm 56.37^{\star \#}$ & $396.68 \pm 55.78^{a^{\star \#}}$ & $431.96 \pm 71.44^{b^{\star \#}}$ & 0.002 \\
\hline \multirow[t]{3}{*}{ UCHL 1} & $\mathrm{~T}_{1}$ & $422.60 \pm 165.27$ & $413.43 \pm 172.77$ & $434.58 \pm 186.98$ & 0.906 \\
\hline & $\mathrm{T}_{2}$ & $828.16 \pm 134.20^{*}$ & $661.96 \pm 166.73^{a^{*}}$ & $782.00 \pm 177.36^{b^{*}}$ & 0.001 \\
\hline & $T_{3}$ & $1223.00 \pm 126.37^{\star \#}$ & $849.21 \pm 175.51^{\mathrm{a}^{\star} \#}$ & $1068.50 \pm 167.71^{a b^{*} \#}$ & $<0.001$ \\
\hline \multicolumn{6}{|c|}{ Data are presented as mean \pm standard deviation $(\mathrm{pg} / \mathrm{mL})$. } \\
\hline \multicolumn{6}{|c|}{ GFAP, glial fibrillary acidic protein; UCHL1, ubiquitin carboxyl-terminal hydrolase isozyme L1. } \\
\hline \multicolumn{6}{|c|}{ a $P<0.05$ compared to Group $A,{ }^{b} P<0.05$ compared to Group B at the same point in time. } \\
\hline \multicolumn{6}{|c|}{${ }^{*} P<0.05,{ }^{\#} P<0.05$ compared to $T_{1}, T_{2}$ within each group, respectively. } \\
\hline
\end{tabular}

\section{Discussion}

Our study investigated effects of intraoperative LPV on respiratory function and the incidences of postoperative complications in emergency TBI patients. The results demonstrated that intraoperative continuous administration of small VT + PEEP could improve oxygenation and respiratory mechanics parameters, decrease incidences of PPCs, and lower increase in posttraumatic serum levels of brain injury markers. However, implementing intermittent RMs might disturb intraoperative cerebral hemodynamics, leading to the fluctuation of ICP.

Small VT ventilation (6-8 $\mathrm{mL} / \mathrm{kg} \mathrm{CBW})$ is now served as the respiratory care standard for ARDS patients in ICU. A consensus has been formed that it is also suitable for patients with healthy lungs in operating room $[9,10]$. An animal experiment showed that small VT ventilation could more effectively promote the oxygenation of rats with brain injury than large VT ventilation [11]. Furthermore, large VT ventilation in TBI patients might be associated with the occurrence of ARDS, and its incidence increased to some extent with the VT [12]. However, a single use of small VT causes periodic alveolar collapse of local lung 
tissues, thus increasing the risk of atelectasis. Interestingly, this adverse effect can be offset by combining PEEP and/or RMs, which would inevitably involve positive pressure ventilation and might adversely affect ICP and CPP [13-15]. However, another study showed that high PEEP $\left(5-15 \mathrm{cmH}_{2} \mathrm{O}\right)$ could improve $\mathrm{PaO}_{2}$ of local brain tissue without affecting ICP and CPP in patients with TBI and ARDS [16]. In summary, the interaction between the lung and the brain poses an important challenge to the ventilation management in TBI patients. An optimal ventilation strategy for TBI patients requires in-depth discussion.

In this study, two groups of TBI patients were treated with LPV: small VT (8 $\mathrm{mL} / \mathrm{kg} \mathrm{CBW})$, intraoperative continuous administration of $5 \mathrm{cmH}_{2} \mathrm{O}$ PEEP. And some were given RMs before opening and after closing the endocranium. Our results showed that intraoperative application of small VT + PEEP or small VT + $\mathrm{PEEP}+\mathrm{RMs}$ improved oxygenation and pulmonary compliance in TBI patients compared to those treated with conventional MV. However, no significant differences in $\mathrm{PaO}_{2}$ and $\mathrm{Cdyn}$ were found between two intervention groups, suggesting that RMs might not provide further improvement on the basis of PEEP. Ppeak, close to airway pressure, reflects the dynamic compliance of respiratory system. Recently, Pplat was recommended as a better predictor of barotrauma and VALI, which was closer to alveolar pressure and reflects the static compliance of respiratory system [17]. Our results showed that patients intervened with LPV had lower Ppeak and Pplat than those receiving conventional MV, suggesting that the implementation of LPV improved respiratory mechanics parameters, and contributed to relieve barotrauma and PPCs. However, there was a rise in $\mathrm{PaCO}_{2}$ at the end of surgery in patients receiving LPV, which may be explained by $\mathrm{CO}_{2}$ retention resulted from reduced periodic alveolar collapse and expansion following small VT. But the little rise could be considered as a compensatory state that would not cause obvious pathological damage [18]. The incidence of intraoperative hypoxemia was lower in two intervention groups, while the cardiovascular adverse reactions in small VT + PEEP + RMs group were more frequently observed. Since intrathoracic pressure increased rapidly in a short time, RMs-treated patients generated a decrease in blood volume returning to the heart, and the cardiac output [13]. Collectively, although small VT + PEEP + RMs can improve intraoperative oxygenation and respiratory mechanics parameters in TBI patients, RMs may cause adverse effects on hemodynamics, while small VT only combined with PEEP can improve lung function without affecting their circulatory stability.

Another primary outcome was the incidence of PPCs in three groups. PPCs are the most common midterm complications after major surgery and strongly linked to clinical prognosis [19]. An observational study in 29 countries named LAS VEGAS showed that $80 \%$ severe TBI patients developed PPCs [20]. Serious pulmonary complications, like ARDS, NPE or VALI, are associated with high mortality, unfavorable neurological outcomes, longer ICU retention and hospital stays in TBI patients [21]. We investigated the incidence of pulmonary complications within postoperative 30 days. The incidences of hypoxemia, pulmonary infection, and atelectasis in LPV-intervened patients were significantly lower than those of patients receiving conventional MV, which was consistent with Marret E et al [22] study. Moreover, using LPV significantly reduced postoperative ventilation time, which might be attributed to the improvement of intraoperative respiratory function and decreased risk of PPCs by small VT combined with PEEP and 
RMs. The GOSE score is usually used to evaluate the degree of disability and neurological prognosis of TBI patients [23]. Our results showed no statistical differences in GOSE score among three groups after 30 days, probably owing to a short follow-up time and limited sample size. Postoperative neurological complications and hospital stays were comparable among three groups, possibly because improving ventilation strategy alone hardly achieved a breakthrough in neurological outcomes of TBI patients. Surgical factors, the quality of nursing, and family economical state, are all needed to be considered comprehensively.

The biggest concern about TBI patients with perioperative LPV is that it may have an adverse effect on ICP and CPP. Ultrasound measurement of ONSD is a novel non-invasive method, widely used to dynamically and rapidly assess changes in ICP. It has a close correlation with canonical direct intubation in ventricle to estimate intracranial hypertension $[24,25]$. Our ultrasound results showed no significant changes in ONSD at any time both in conventional MV and small VT + PEEP group, suggesting that intraoperative continuous administration of $5 \mathrm{cmH}_{2} \mathrm{O}$ PEEP did not affect patients' ICP. Mascia et al [26] randomly applied $5 \mathrm{cmH}_{2} \mathrm{O}$ or $10 \mathrm{cmH}_{2} \mathrm{O}$ PEEP in 12 patients along with brain injury and ARDS. They found that the PEEP level, which was insufficient to give rise to excessive alveolar expansion or an evident increase in $\mathrm{PaCO}_{2}$, had no significant impact on ICP, and could safely improve oxygenation. Likewise, if the PEEP value was lower than that of ICP during MV, the elevation of intrathoracic pressure within a certain range would not increase ICP [27]. Therefore, $5 \mathrm{cmH}_{2} \mathrm{O}$ PEEP was applied in our study not lifting ICP visibly, because it did not cause alveolar hyperinflation or it was less than patients' ICP. However, RMs could rapidly expand the alveoli in a short period and increase the pressure in the thoracic cavity, which blocked the return of systemic circulation to the right atrium (cerebral venous reflux) and eventually increased ICP. After ceasing RMs, the intrathoracic pressure dropped to normal, and patient's ICP decreased accordingly [28]. Consistent with previous finding [29], a single RM transiently increased ONSD, which returned to the baseline within 5-10 minutes, indicating that RMs had the risk of elevating ICP of TBI patients.

Immediately after acute brain injury, astrocytes undergo mechanical deformation or local necrosis, leading to increased serum concentration of GFAP [30-32]. UCHL1 is a neuron-specific cytosolic enzyme and its serum level in acute phase of cerebral injury strongly correlated with the severity of damage [33]. Combined testing of GFAP and UCHL1 could more accurately diagnose the severity of brain damage and predict the long-term prognosis [34]. Here, postoperative serum levels of GFAP and UCHL1 in each group were higher than before. Possibly because they were released from necrotic cells and accumulated with the posttraumatic time course [35]. Furthermore, both serum levels of GFAP and UCHL1 in Group B were lower than the others at $T_{3}$. It is considered that PEEP can improve oxygenation, reduce the release of inflammatory mediators of the lung and brain, thereby alleviating secondary injuries. Interestingly, additional RMs posed a large continuous positive airway pressure in a short time, which had an adverse effect on cerebral hemodynamics that neutralized favorable results of implementing PEEP. Hence, intraoperative application of small VT + PEEP could prevent further brain damage in TBI patients to some extent. Whether RMs played the same role remained further exploration. 
Several limitations in our study should be noteworthy. First, actual values and accurate changes in ICP were unable to be obtained from ultrasound measurement of ONSD. Second, our results may not be applicable to other neurosurgical patients, like intracranial tumors, craniocerebral injury in sitting position during operation, spontaneous cerebral hemorrhage, etc. Third, during the study period, especially before operation, some severe patients were treated with mannitol and dexamethasone due to their conditions, which might affect the results consequently. Fourth, a 30-day postoperative follow-up was unable to accurately evaluate long-term survival and life quality of TBI patients. Last, it was a single-center study with limited sample size. Large-scale clinical trials are needed in the future to validate the impact of intraoperative LPV on TBI patients.

\section{Conclusions}

Intraoperative continuous administration of small VT + PEEP is beneficial to TBI patients, manifesting as improved oxygenation and respiratory mechanics parameters, decreased incidences of PPCs, and lowered increase in posttraumatic serum levels of brain injury markers. However, additional RMs should be cautiously applied in these patients, since it is prone to disturbing intraoperative cerebral hemodynamics.

\section{Abbreviations}

TBI

Traumatic brain injury; NPE:Neurogenic pulmonary edema; VALI:Ventilator-associated lung injury; VT:Tidal volume; CBW:Corrected body weight; LPV:Lung-protective ventilation; PEEP:Positive endexpiratory pressure; Pplat:Airway plateau pressure; ARDS:Acute respiratory distress syndrome; ICU:Intensive care unit; MV:Mechanical ventilation; PPCs:Postoperative pulmonary complications; RMs:Recruitment maneuvers; ICP:Intracranial pressure; CPP:Cerebral perfusion pressure; BMI:Body mass index; ASA:American Society of Anesthesiologists; $\mathrm{P}_{\mathrm{ET}} \mathrm{CO}_{2}$ :End-tidal carbon dioxide partial pressure; $\mathrm{SpO}_{2}$ :Oxygen saturation; $\mathrm{PaO}_{2}$ :Arterial partial pressure of oxygen; $\mathrm{PaCO}_{2}$ :Arterial partial pressure of carbon dioxide; Cdyn:Pulmonary dynamic compliance; Ppeak:Airway peak pressure; HR:Heart rate; MAP:Mean arterial pressure; SBP:Systolic blood pressure; GFAP:Glial fibrillary acidic protein; UCHL1:Ubiquitin carboxyl-terminal hydrolase isozyme L1; ONSD:Optic nerve sheath diameter; GOSE:Glasgow Outcome Scale Extended; GCS:Glasgow Coma Scale

\section{Declarations}

\section{Acknowledgements}

Not applicable.

\section{Authors' contributions}


JG, LLJ and YJW have given substantial contributions to the conception and design of the manuscript, $\mathrm{YZ}, \mathrm{DHL}$ and KSY to acquisition, analysis and interpretation of the data. All authors have participated in drafting the manuscript, JG and LLJ revised it critically. All authors read and approved the final manuscript.

\section{Funding}

None.

\section{Availability of data and materials}

The datasets used and/or analyzed during the current study are available from the corresponding author on reasonable request.

\section{Ethics approval and consent to participate}

This study was approved by the Ethical Committee of Northern Jiangsu People's Hospital (2019113). All procedures performed involving human participants were in accordance with the ethical standards of the Declaration of Helsinki 1964 and its later amendments. Informed consent was obtained from all individual participants included in the study or their relatives.

\section{Consent for publication}

Not applicable.

\section{Competing interests}

The authors declare that they have no competing interests.

\section{References}

1. Maas A, Menon DK, Adelson PD, Andelic N, Bell MJ, Belli A, et al. Traumatic brain injury: integrated approaches to improve prevention, clinical care, and research. Lancet Neurol. 2017;16(12):9871048.

2. Zygun DA, Kortbeek JB, Fick GH, Laupland KB, Doig CJ. Non-neurologic organ dysfunction in severe traumatic brain injury. Crit Care Med. 2005;33(3):654-60.

3. Koutsoukou A, Katsiari M, Orfanos SE, Kotanidou A, Daganou M, Kyriakopoulou M, et al. Respiratory mechanics in brain injury: A review. World J Crit Care Med. 2016;5(1):65-73.

4. Lopez-Aguilar J, Quilez ME, Marti-Sistac O, Garcia-Martin C, Fuster G, Puig F, et al. Early physiological and biological features in three animal models of induced acute lung injury. Intensive Care Med. 2010;36(2):347-55.

5. Fan E, Brodie D, Slutsky AS. Acute Respiratory Distress Syndrome: Advances in Diagnosis and Treatment. JAMA. 2018;319(7):698-710. 
6. Boone MD, Jinadasa SP, Mueller A, Shaefi S, Kasper EM, Hanafy KA, et al. The Effect of Positive EndExpiratory Pressure on Intracranial Pressure and Cerebral Hemodynamics. Neurocrit Care. 2017;26(2):174-81.

7. Schirmer-Mikalsen K, Moen KG, Skandsen T, Vik A, Klepstad P. Intensive care and traumatic brain injury after the introduction of a treatment protocol: a prospective study. Acta Anaesthesiol Scand. 2013;57(1):46-55.

8. Sun ZQ. Estimation of Sample Size. In: Sun ZQ, Xu YY, Ma J, editors. Medical Statistics. 4th ed. Beijing: People's Medical Publishing House; 2014. p. 573-83.

9. Futier E, Constantin JM, Paugam-Burtz C, Pascal J, Eurin M, Neuschwander A, et al. A trial of intraoperative low-tidal-volume ventilation in abdominal surgery. N Engl J Med. 2013;369(5):428-37.

10. O'Gara B, Talmor D. Perioperative lung protective ventilation. BMJ. 2018;362:k3030.

11. Krebs J, Tsagogiorgas C, Pelosi P, Rocco PR, Hottenrott M, Sticht C, et al. Open lung approach with low tidal volume mechanical ventilation attenuates lung injury in rats with massive brain damage. Crit Care. 2014;18(2):R59.

12. Mascia L, Zavala E, Bosma K, Pasero D, Decaroli D, Andrews $P$, et al. High tidal volume is associated with the development of acute lung injury after severe brain injury: an international observational study. Crit Care Med. 2007;35(8):1815-20.

13. Ruggieri F, Beretta L, Corno L, Testa V, Martino EA, Gemma M. Feasibility of Protective Ventilation During Elective Supratentorial Neurosurgery: A Randomized, Crossover, Clinical Trial. J Neurosurg Anesthesiol. 2018;30(3):246-50.

14. Georgiadis D, Schwarz S, Baumgartner RW, Veltkamp R, Schwab S. Influence of positive endexpiratory pressure on intracranial pressure and cerebral perfusion pressure in patients with acute stroke. Stroke. 2001;32(9):2088-92.

15. Bein T, Kuhr LP, Bele S, Ploner F, Keyl C, Taeger K. Lung recruitment maneuver in patients with cerebral injury: effects on intracranial pressure and cerebral metabolism. Intensive Care Med. 2002;28(5):554-8.

16. Nemer SN, Caldeira JB, Santos RG, Guimaraes BL, Garcia JM, Prado D, et al. Effects of positive endexpiratory pressure on brain tissue oxygen pressure of severe traumatic brain injury patients with acute respiratory distress syndrome: A pilot study. J Crit Care. 2015;30(6):1263-6.

17. Chan MC, Tseng JS, Chiu JT, Hsu KH, Shih SJ, Yi CY, et al. Prognostic value of plateau pressure below 30 cm H2O in septic subjects with acute respiratory failure. Respir Care. 2015;60(1):12-20.

18. Barnes T, Zochios V, Parhar K. Re-examining Permissive Hypercapnia in ARDS: A Narrative Review. Chest. 2018;154(1):185-95.

19. Jammer I, Wickboldt N, Sander M, Smith A, Schultz MJ, Pelosi P, et al. Standards for definitions and use of outcome measures for clinical effectiveness research in perioperative medicine: European Perioperative Clinical Outcome (EPCO) definitions: a statement from the ESA-ESICM joint taskforce on perioperative outcome measures. Eur J Anaesthesiol. 2015;32(2):88-105. 
20. Epidemiology, practice of ventilation and outcome for patients at increased risk of postoperative pulmonary complications: LAS VEGAS - an observational study in 29 countries. Eur J Anaesthesiol. 2017;34(8):492-507.

21. Steyerberg EW, Wiegers E, Sewalt C, Buki A, Citerio G, De Keyser V, et al. Case-mix, care pathways, and outcomes in patients with traumatic brain injury in CENTER-TBI: a European prospective, multicentre, longitudinal, cohort study. Lancet Neurol. 2019;18(10):923-34.

22. Marret E, Cinotti R, Berard L, Piriou V, Jobard J, Barrucand B, et al. Protective ventilation during anaesthesia reduces major postoperative complications after lung cancer surgery: A double-blind randomised controlled trial. Eur J Anaesthesiol. 2018;35(10):727-35.

23. Zelnick LR, Morrison LJ, Devlin SM, Bulger EM, Brasel KJ, Sheehan K, et al. Addressing the challenges of obtaining functional outcomes in traumatic brain injury research: missing data patterns, timing of follow-up, and three prognostic models. J Neurotrauma. 2014;31(11):1029-38.

24. Whiteley JR, Taylor J, Henry M, Epperson TI, Hand WR. Detection of elevated intracranial pressure in robot-assisted laparoscopic radical prostatectomy using ultrasonography of optic nerve sheath diameter. J Neurosurg Anesthesiol. 2015;27(2):155-9.

25. Cammarata G, Ristagno G, Cammarata A, Mannanici G, Denaro C, Gullo A. Ocular ultrasound to detect intracranial hypertension in trauma patients. J Trauma. 2011;71(3):779-81.

26. Mascia L, Zavala E, Bosma K, Pasero D, Decaroli D, Andrews P, et al. High tidal volume is associated with the development of acute lung injury after severe brain injury: an international observational study. Crit Care Med. 2007;35(8):1815-20.

27. Chen H, Menon DK, Kavanagh BP. Impact of Altered Airway Pressure on Intracranial Pressure, Perfusion, and Oxygenation: A Narrative Review. Crit Care Med. 2019;47(2):254-63.

28. Nemer SN, Caldeira JB, Azeredo LM, Garcia JM, Silva RT, Prado D, et al. Alveolar recruitment maneuver in patients with subarachnoid hemorrhage and acute respiratory distress syndrome: a comparison of 2 approaches. J Crit Care. 2011;26(1):22-7.

29. Lyon M, Agrawal P, Friez K, Gordon R, Morales I, Fang ZL, et al. Effect of History of Mild Traumatic Brain Injury on Optic Nerve Sheath Diameter Changes after Valsalva Maneuver. J Neurotrauma. 2018;35(4):695-702.

30. Nylen K, Ost M, Csajbok LZ, Nilsson I, Blennow K, Nellgard B, et al. Increased serum-GFAP in patients with severe traumatic brain injury is related to outcome. J Neurol Sci. 2006;240(1-2):85-91.

31. Czeiter E, Mondello S, Kovacs N, Sandor J, Gabrielli A, Schmid K, et al. Brain injury biomarkers may improve the predictive power of the IMPACT outcome calculator. J Neurotrauma. 2012;29(9):1770-8.

32. McMahon PJ, Panczykowski DM, Yue JK, Puccio AM, Inoue T, Sorani MD, et al. Measurement of the glial fibrillary acidic protein and its breakdown products GFAP-BDP biomarker for the detection of traumatic brain injury compared to computed tomography and magnetic resonance imaging. $J$ Neurotrauma. 2015;32(8):527-33.

33. Li J, Yu C, Sun Y, Li Y. Serum ubiquitin C-terminal hydrolase L1 as a biomarker for traumatic brain injury: a systematic review and meta-analysis. Am J Emerg Med. 2015;33(9):1191-6. 
34. Mondello S, Jeromin A, Buki A, Bullock R, Czeiter E, Kovacs N, et al. Glial neuronal ratio: a novel index for differentiating injury type in patients with severe traumatic brain injury. J Neurotrauma. 2012;29(6):1096-104.

35. Papa L, Brophy GM, Welch RD, Lewis LM, Braga CF, Tan CN, et al. Time Course and Diagnostic Accuracy of Glial and Neuronal Blood Biomarkers GFAP and UCH-L1 in a Large Cohort of Trauma Patients With and Without Mild Traumatic Brain Injury. JAMA Neurol. 2016;73(5):551-60.

\section{Figures}

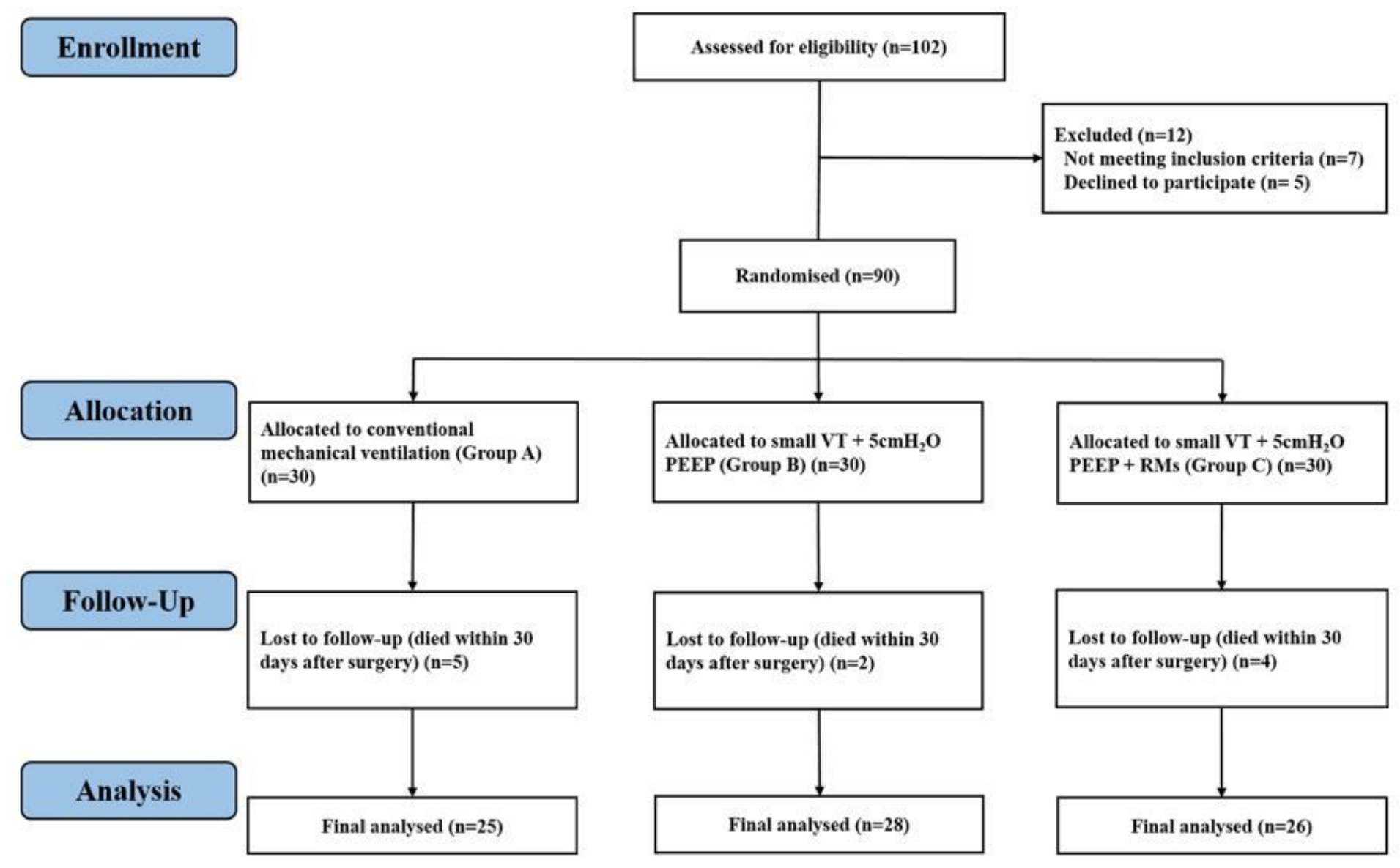

Figure 1

Consolidated Standards of Reporting Trials (CONSORT) flow diagram. PEEP, positive end-expiratory pressure; RMs, recruitment maneuvers; VT, tidal volume. 


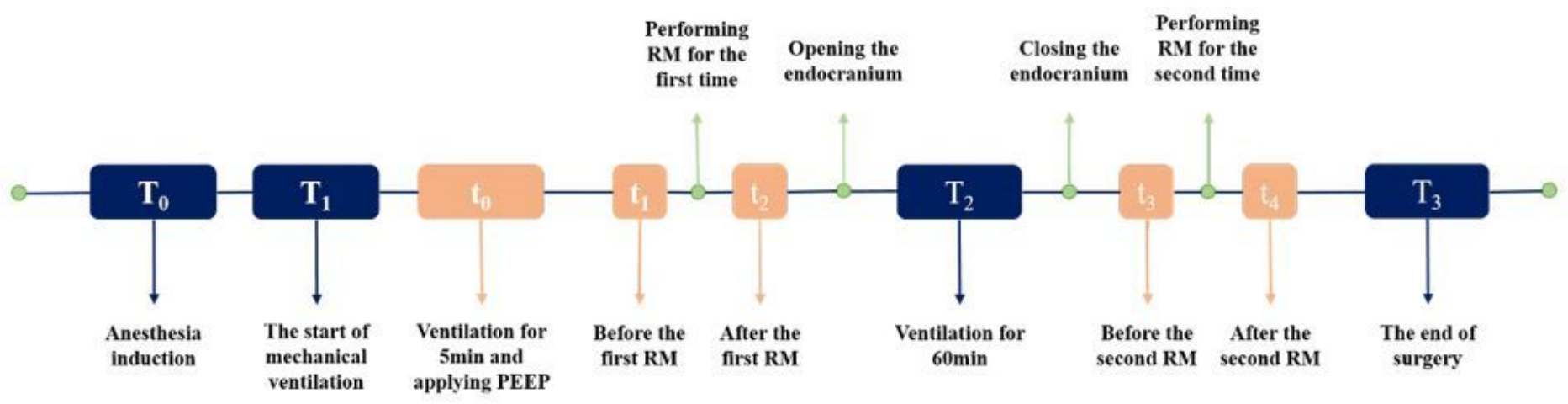

Figure 2

Timeline of intraoperative lung-protective ventilation strategy implementation. PEEP, positive endexpiratory pressure; RM, recruitment maneuver. 